\title{
Study on the Release of Vancomycin-Loaded Calcium Sulphate by Three-Dimensional Printing in Vitro
}

\author{
Qian Zhang ${ }^{1}$, Huailong Sun ${ }^{1}$
}

\begin{abstract}
${ }^{1}$ Department of Special Medicine, Basic Medical college ,308 Ningxia Road, Qingdao University, QingDao, China, 266071

Abstract: Objective: To prepare a three-layer cylindrical drug carrier with different drug concentration in each layer by using 3D printing technology, and compare it with the drug carrier prepared by ordinary mold to discuss the drug release rules of the two groups of carriers. Methods: the experimental group was the three layers of cylindrical calcium sulfate carrier prepared by the 3D printer, vancomycin content is different, each layer from top to bottom respectively contain vancomycin $25 \mathrm{mg}, 50 \mathrm{mg}, 25 \mathrm{mg}$, compared with vancomycin and calcium sulfate by mould preparation after mixing, each contain vancomycin $100 \mathrm{mg}$, two groups of total content of vancomycin was same, Soak in $5 \mathrm{ml}$ PBS buffer after drying, respectively at 1, 3, 5, 7, 10,14, 18, 22, 26, 30day point in time, detection of drug release from two groups of carriers ,and discuss the drug release rule of two groups. Results: The drug release of the experimental group was more stable than that of the control group. Conclusion: The release effect of sustained release vector prepared by $3 \mathrm{D}$ printing is better than that prepared by ordinary mold.
\end{abstract}

Keywords: 3D Printing, Infectious Bone Defect, Vancomycin-Loaded Calcium Sulphate, Sustained-Release Vector

\section{Introduction}

The treatment of chronic osteomyelitis is a difficult clinical problem. Routine treatment includes thorough debridement, continuous avage and intravenous antibiotics for 4-6 weeks ${ }^{[1]}$. the treatment time is long and the cost is high. In recent years, a mixture of vancomycin and calcium sulfate(Vancomycin-loaded Calcium Sulphate), has been widely used in the experiment and Clinic. The Vancomycin-loaded Calcium Sulphate can release high concentration of vancomycin in the local lesion, while the blood concentration is low, the safety is high, and and has the bone guide function, which fully meets the requirements of the treatment of osteomyelitis ${ }^{[2]}$. Currently,the common preparation methods was vancomycin and calcium sulfate are mixed into granules in a mold, but the drug release Is not controllable. In this experiment, 3D printing technology was used to prepare a three-layer cylindrical drug release carrier with different drug concentration, which was compared with the artificialy prepared carrier to analyze the drug release law.

\section{Methods}

1.1 Preparation of control group carrier

According to distilled water : vancomycin hydrochloride: anhydrous calcium sulfate $6 \mathrm{ml}: 1 \mathrm{~g}: 9 \mathrm{~g}$ of proportioning, mixing evenly, with $5 \mathrm{ml}$ syringe as mould, excision of the front end of the syringe, in front of the mixture solidified into the mold, after being dry, made $13 \mathrm{~mm}$ cylindrical structure, weighing after drying, and cut to spare part to each weighs $1.0 \mathrm{~g}$, the height after cutting is $9 \mathrm{~mm}$, each contain vancomycin $100 \mathrm{mg}$, preparing five samples, and operating in a sterile environment.
1.2 Preparation of vancomycin sulfate by 3D printing
Dissolve vancomycin hydrochloride in water, add A small amount of binder, spread powder on the workbench, and set parameters in the control system, each layer of 200 microns, layered overlay, diameter of $13 \mathrm{~mm}$, from top to bottom is divided into A, B, C three layers, each layer height $3 \mathrm{~mm}$, contain vancomycin is $25 \mathrm{mg}$, $50 \mathrm{mg}$ and $25 \mathrm{mg}$ respectively, the content of vancomycin in each carrier is $100 \mathrm{mg}$, preparation of five. 


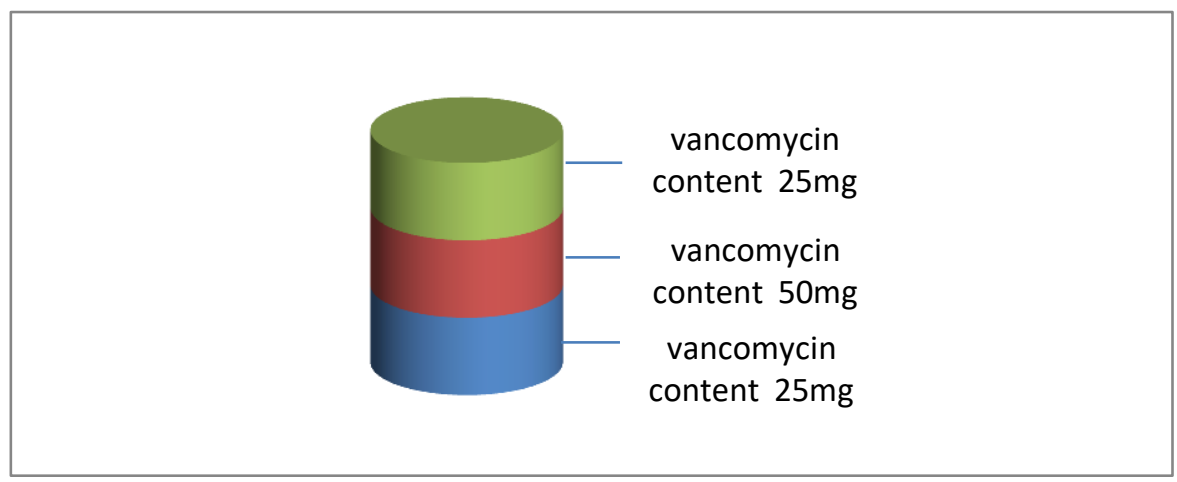

Fig. 1 structure of 3D pr inted drug delivery carrier

\subsection{Detection methods}

Determination of vancomycin release. Put two sets of a total of 10 samples respectively in $10 \mathrm{ml}$ centrifuge tube, add 5ml PBS buffer immersion. Replacement of liquids and detection of drug concentration on day1, 3 , $5,7,10,14,18,22,26,30$, respectively. With high performance iquid chromatograph (SHMADZU LC-2030 CN), chromatographic column: Inertsil ODS -SP (4.6 X150mm'5 microns), mobile phase: methanol
$-0.05 \mathrm{~mol} / \mathrm{L}$ of potassium dihydrogen phosphate buffer(PH3.0) $=20: 8$, The flow rate was $1.0 \mathrm{ml} / \mathrm{min}$, column temperature was $30^{\circ} \mathrm{C}$, detection wavelength was $236 \mathrm{~nm}$, and injection volume was $20 \mu \mathrm{L}$.

\section{Results}

2.1 The average release concentration of the two carriers at each time point is shown in Table 1.

Table 1 The average release concentration at each time point of the two carriers

$\mathrm{mg} / \mathrm{mL}$

\begin{tabular}{ccccccccccc}
\hline \multirow{2}{*}{ group } & 1 & 3 & 5 & 7 & 10 & 14 & 18 & 22 & 26 & 30 \\
\cline { 2 - 11 } & 1 & 2.95 & 2.18 & 1.61 & 1.05 & 0.86 & 1.07 & 1.54 & 0.91 & 0.39 \\
\hline $\begin{array}{c}\text { Experience } \\
\text { group }\end{array}$ & 3.72 & 2.07 & 1.95 & 1.11 & 0.57 & 0.23 & 0.15 & 0.06 & 0.02 \\
\hline $\begin{array}{c}\text { Control } \\
\text { group }\end{array}$ & 5.85 & 3.82 & 2.78 & 1.95 \\
\hline
\end{tabular}

Are Visible in table 1, the initial drug release of the two carriers was very high, gradual stabilization in the medium term, sustained drug release more than 30 days, the experimental group initial concentration of drug release, although local antimicrobial effect has reached, but was lower than those of control group to release a quantity, the medium-term has been able to maintain at a high drug release effect, on the 22nd day, the drug release increased compared with the previous time poin, and then gradually reduced.

2.2 The cumulative release rate- time curve of vancomycin of the two groups were shown In figure 1

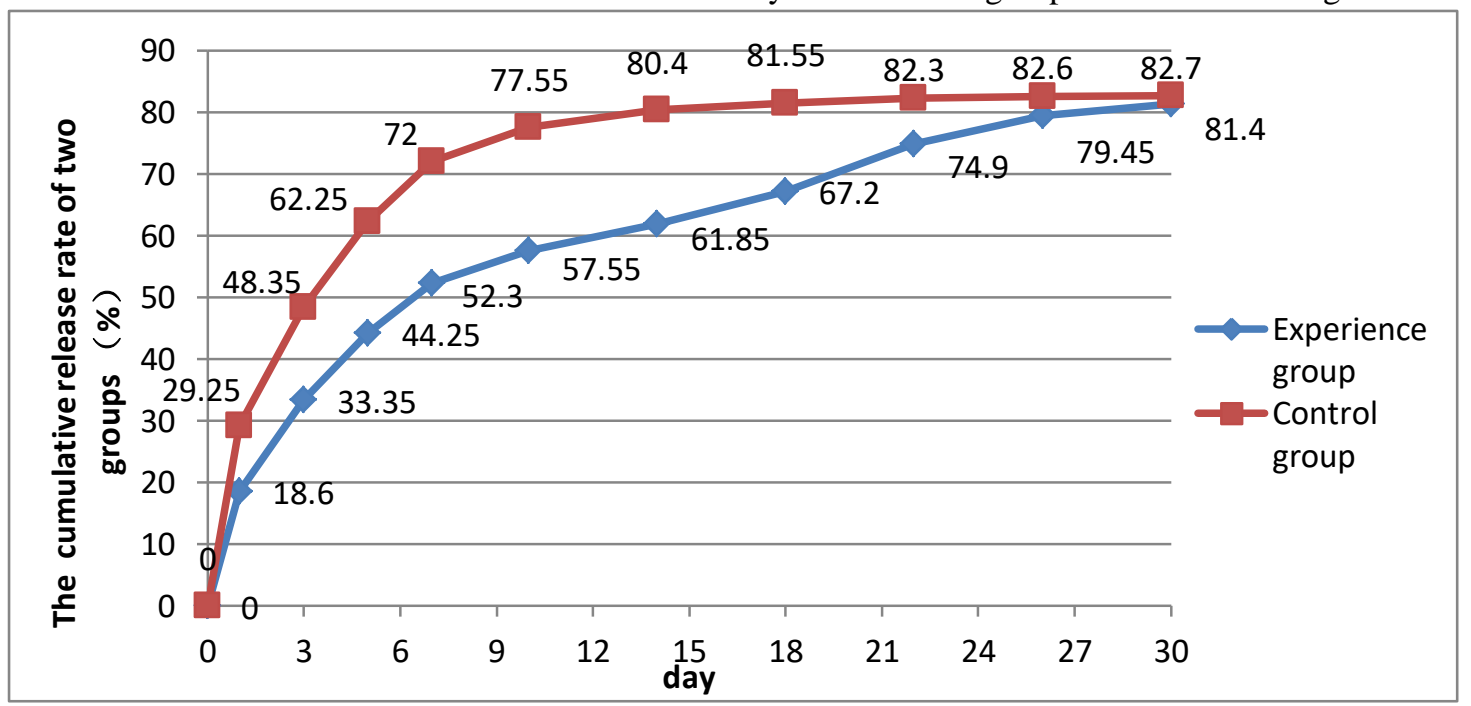

Fig. 2 Cumulative drug release rate-time curves of two groups of carriers 
As shown in figure 2, the cumulative release rate of the control group was $82.7 \%$, and the cumulative release rate of the experimental group was $81.4 \%$. The drug release law of the control group basically conforms to the Higuchi equation, and the $3 \mathrm{~d}$-printed carrier is more stable than that of the control group, the drug release in the middle stage can also be maintained at a higher level, and the antibacterial effect can be maintained for a longer time.

\section{Discussion}

The treatment of chronic osteomyelitis is based on thorough debridement, but it is difficult to ensure the absolute cleanliness of debridement in practice. Therefore, the application of carrier loaded antibiotics with slow release function is an ideal choice ${ }^{[3]}$. However, 3d printiing technology can accurately prepare drug-oaded artificial bone with complex structure, and can arrange multiple drugs arbitrarily. The rip-inh-controlled release drug prepared by $\mathrm{Wu}$ weigang et al. ${ }^{[4]}$ with $3 \mathrm{~d}$ printing technology has the characteristics of controlled release and sustained release of drugs, realizing the sequential release of both drugs in the experiment. This experiment was using the 3D printing technology to prepare a three-layer cylindrical carrier, and the drug release law was affected by the different drug concentrations of the three layers, indicating that the controlled drug release effect of the carrier could be achieved by setting the drug content of each part of the carrier.

\section{Reference}

1. Hatzenbuehler J,Pulling TJ.Diagnosis and management of osteomyelitis.Am Fam Physician,2011,84(9):1027-1033.

2. ZHANG Z, ZHANG C, GUO Q F, et al. Application of Vancomycin-loaded Calcium Sulphate in Treatment of Osteomyelitis [J]. ACTA ACADEMIAE MEDICINAE SINICAE,2013,35(03):337-342.

3. Li G, Ning X R, Chen T J, et al.A contrast study on bone graft substitutes loaded with vancomycin in the treatment for chronic osteomylitis [J]. Medical Journal of National Defending Forcesin Southwest China,2014,24(04):367-369.

4. WUWei-Gang ZHENGQi-Xin GUOXiao-Dong. Experimental Study of a Rifampicine-isoniazid-controlled Release Drug Implant [J]. Chinese Journal of Biomedical Engineering,2010,29(01):137-143. 